${ }^{3}$ Levy D, Russell WE, Middlebrook G. Dosages of isonazid and streptomycin for routine chemotherapy of tuberculosis. Tubercle 1960;41:23-31.

${ }^{4}$ Palmer WR, Höffler D. Carbenicillin. Basisdaten zur Therapie mit einem Antibioticum. München, Wien, Baltimore: Urban und Schwarzenberg, 1977.

${ }^{5}$ Sutherland R, Rolinson GN. Penicillins. In: Reeves DS, Phillips $\mathrm{I}$, Williams JD, Wire R, eds. Laboratory methods in antimicrobial chemotherapy. Edinburgh, London and New York: Churchill-Livingstone, 1978:171-8.

${ }^{\circ}$ Fabius GThJ, Mouton RP. Rapid microbiological blood level determination of aminoglycoside antibiotics. In: Mouton RP, ed. Aminoglycoside assays, methods and clinical relevance. Amsterdam, Oxford: Excerpta Medica, 1979:12-22.

${ }^{7}$ Edberg SC, Bottonbley $\mathrm{CJ}$ and Gam K. Use of sodium polyanethol sulfonate to selectively inhibit aminoglycoside and polymyxin antibiotics in a rapid blood level antibiotic assay. Antimicrob Agents Chemother 1976;9/3:414-7.

Requests for reprints to: Dr BH Postma, Laboratory for Medical Microbiology, Herman Gorterlaan 2, 5644 SW Eindhoven, The Netherlands.

\section{Congo blue: a rapid stain for elastic fibres}

\author{
JD DAVIES, * EW YOUNG $\dagger$ University Department of \\ Pathology, Bristol Royal Infirmary, Bristol \\ BS2 $8 H W$
}

There are numerous empirical methods for staining elastic fibres. ${ }^{1}$ Most require relatively lengthy procedures which involve overnight incubation, and are therefore not adapted to simultaneous evaluation with the corresponding haematoxylin and eosinstained sections.

Congo red, a dye which was initially used in commercial colouring of cotton fabrics, is often employed for the diagnosis of amyloid in human tissue. ${ }^{23}$ Most batches of Congo red, even when used in the standardised saturated salt conditions described by Puchtler et al $^{4}$ also stain elastic fibres. Such elastic staining can, of course, be a disadvantage when identification of amyloid is desired. Although the anisotropic and dichroic characteristics of amyloid usually distinguish it from elastic fibres, the inexperienced histopathologist may find the distinction difficult. This problem may be accentuated in the dermis, where the recent demonstration of the $\mathbf{P}$ component of amyloid in elastic fibres 5 could well add to the confusion.

The Congo staining method which we describe is a modification of that of Highman. ${ }^{3}$ It demonstrates elastic fibres clearly and distinguishes them sharply from amyloid.

\section{Material and methods}

The tissues were fixed in unbuffered fresh formol saline. They were processed via xylene for paraffin embedding in the routine manner. Paraffin sections ( $5 \mu \mathrm{m})$ were taken to water.

The sections were stained with $0.5 \%$ (wt/vol) Congo red in $50 \%$ alcohol according to the method of Highman $^{3}$ for $10 \mathrm{~min}$. Thereafter the sections were rinsed in distilled water.

Instead of the exposure to alkaline ethanol, appropriate for Highman's Congo red procedure, the sections are then rinsed for about $10 \mathrm{~s}$ in $1 \%$ hydrochloric acid in $70 \%$ ethanol. With this treatment the sections become blue to the naked eye.

\footnotetext{
*Address for correspondence: Dr JD Davies, University Department of Pathology, Level 9 Phase I, Bristol Royal Infirmary, Bristol BS2 8HW.

$\dagger$ Present address: Department of Neuropathology, Frenchay Hospital, Bristol BS16 1LE.
}

Accepted for publication 24 February 1982 
The sections are then flooded with absolute alcohol until they appear pink macroscopically. This procedure requires only 10 to $20 \mathrm{~s}$.

Thereafter the sections are cleared in xylene and mounted in DPX before application of glass cover slips.

\section{Results}

Elastic fibres arê stäined deep blue. Their intensity of staining is considerably enhanced by comparison with conventional Congo red staining (Figure). Amyloid stains red, although this colour is rather more mauve than in alkaline Congo red procedures. A green dichroism is readily demonstrated in the amyloid when viewed in polarised light after this staining method. Other tissue components are stained in shades of red and blue, although none are as intensely stained as amyloid and elastic fibres. Nuclei are stained blue, and therefore require no counterstaining. The cytoplasm of granulocytes, macrophages and Paneth cells stain blue. Muscle cytoplasm stains a moderate red.

Prolonged treatment with absolute alcohol in the $\stackrel{\vec{F}}{\stackrel{\vec{F}}{+}}$ stage before clearing will alter the staining. If it is taken beyond the appearance of a macroscopic pink colour, this will eventually lead to conversion of all $\overrightarrow{\widetilde{D}}$ "Congo blue" staining to red in the microscopic preparations.

The use of acid alcohol is not obligatory. Aqueous $\vec{\circ}$ solutions of hydrochloric acid will effect the same colour changes. At $\leqslant \mathrm{pH} 1 \cdot 0$, aqueous hydrochloric $\vec{\omega}$ acid will quickly convert all Congo-staining tissue elements to blue. At pH $2 \cdot 0$ the selective red and blue coloration described above, using $1 \%$ hydro- $\omega$ chloric acid in $70 \%$ ethanol, is produced. With solu- $-v$ tions of $\geqslant \mathrm{pH} 3.0$ no blue coloration is seen.

The final dehydration in absolute alcohol (10 to $20 \mathrm{~s}$ ) must be relatively brief before the clearing in 5 xylene. If the sections are immersed overnight in alcohol before being cleared and mounted, the blue staining of the elastic fibres is unstable and reverts to red.

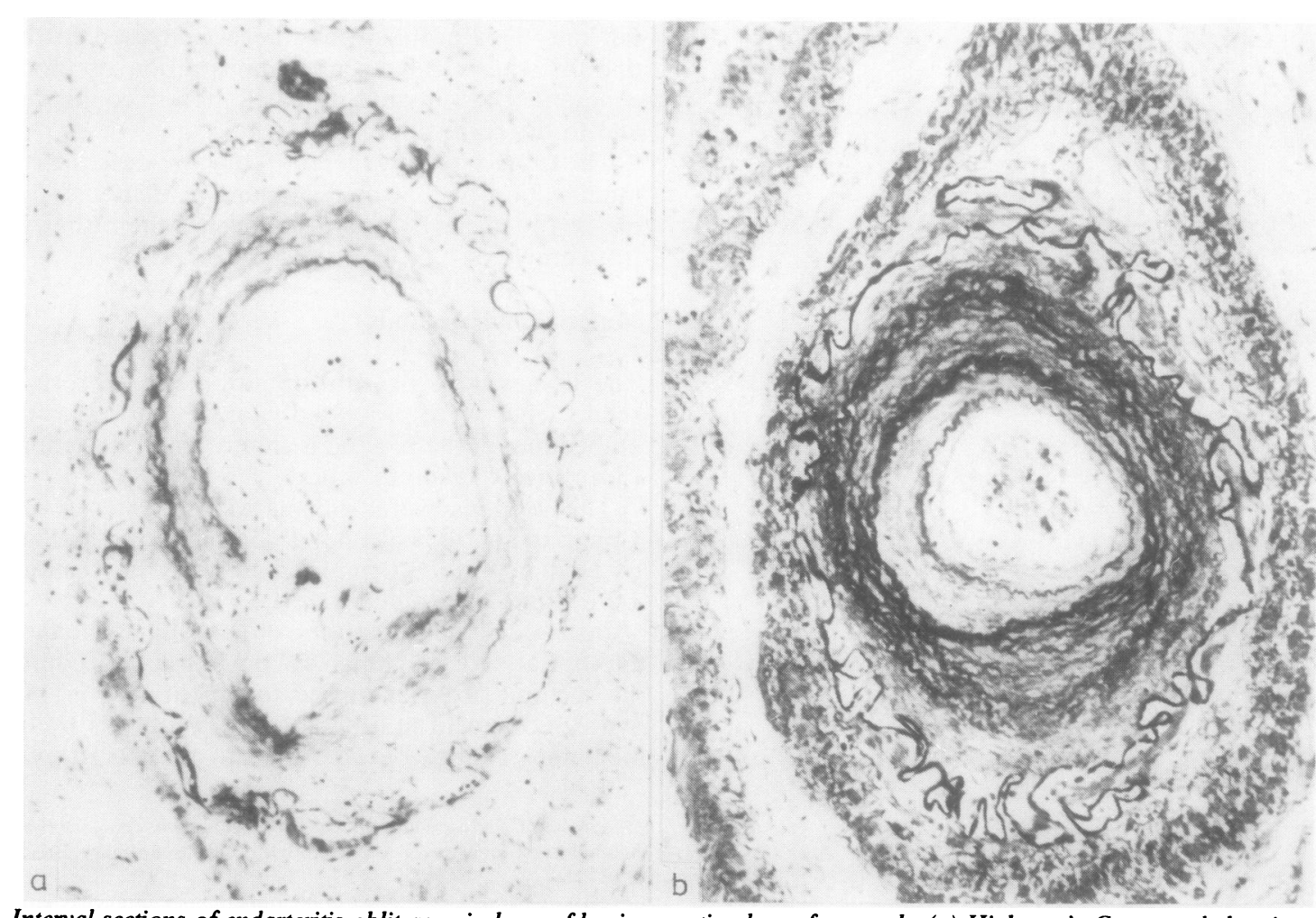

Interval sections of endarteritis obliterans in base of benign peptic ulcer of stomach. (a) Highman's Congo red showing relatively weak red staining elastic-fibres of internal elastic lamina and intima. (b) "Congo-blue": deeper and more extensive staining of intimal, internal elastic lamina and adventitial elastic fibres. $\times 120$. 


\section{Comment}

This method provides a clear distinction between Congo-stainable amyloid and elastic fibres. We have stained elastic fibres in this way in dermis, breast, aorta, the gastrointestinal tract and in veins and arteries from many other organs.

Occasionally the distinction between amyloid and other connective-tissue constituents is not always clear using Congo or Sirius reds. This method helps in their separation, and its rapidity obviates the necessity for overnight staining which is a requirement for many of the elastic-fibre stains in routine diagnostic use. The elimination of lengthy elastic staining is especially advantageous in laboratories where elastic fibres in breast biopsies ${ }^{6}$ are stained routinely. The procedure allows haematoxylin and eosin sections to be examined concurrently with elastic-stained preparations without delay. Unfortunately immunochemical demonstration of elastin in frozen $^{7}$ or fixed paraffin sections ${ }^{8}$ are currently not adapted to such rapid processing.

The histochemical basis of this Congo blue stain is currently obscure. The dye Congo red is an indicator, turning blue in acid conditions. To our knowledge the only previous use of Congo red in acid solutions as a stain for elastic fibres was that described in 1925 by Matsuura. ${ }^{9}$ He used phos- phomolybdic acid in dilute alcohol instead of the hydrochloric acid-alcohol which we have described. His results were similar, although the distinction of elastic from amyloid was not investigated.

We are grateful to Mr SL Mera for helpful discussion of the method, and to Mrs AR Nelson for typing the manuscript.

\section{References}

' Horobin RW, James NT. The staining of elastic fibres with Direct Blue 152. Histochemie 1970;22:324-36.

${ }^{2}$ Tribe CR, Perry VA. Diagnosis of amyloidosis. ACP Broadsheet 1979;92:1-9.

${ }^{3}$ Highman B. Improved methods for demonstrating amyloid in paraffin sections. Archives of Pathology 1946;41:559-62.

${ }^{4}$ Puchtler H, Sweat F, Levine M. On the binding of Congo Red by amyloid. J Histochem Cytochem 1962;10:355-64.

${ }^{5}$ Breathnach SM, Melrose SM, Bhagal B, et al. Amyloid component is located on elastic fibre microfibrils in normal human tissue. Nature 1981;293:652-4.

- Davies JD. Hyperelastosis, obliteration and fibrous plaques in major ducts of the human breast. J Pathol 1973;110:13-26.

${ }^{7}$ McCullagh KG, Barnard K, Davies JD, Partridge SM. Newly synthesised elastin is on the surface of tumour cells in human mammary carcinoma. Experientia 1980;36:1315-6.

${ }^{8}$ Davies JD, Barnard K, Young EW. Peroxidase-antiperoxidase immunolocalisation of newly formed elastin in paraffinembedded breast lesions. Proc 143rd Meeting Path Soc 1981;17-18.

${ }^{9}$ Matsuura S. Über die Färbung mit Kongorot. Folia Anat Jpn 1925;3:107-10.

\section{Letters to the Editor}

Formalised yolk sac antigen in early diagnosis of Legionnaires' disease caused by Legionella pneumophila serogroup 1

Taylor and Harrison ${ }^{1}$ reported that because of the specificity of the formalised yolk sac antigen (FYSA) they were able to examine sera for the presence of antibody to Legionella pneumophila serogroup 1 by the indirect fluorescent antibody test (IFAT) starting at a dilution of 16 with only $3 \%$ of patients with illness other than Legionella infection showing detectable antibody and this only at a dilution of 16 . They further noted that antibody detected at this level could be an early indication of Legionella infection. In view of their observation and since the submission of our report, ${ }^{3}$ we have examined 24 sera from cases of Legionella infection taken early in the course of illness and which had been found to be negative at a dilution of 32 in the IFAT using heat-killed polyvalent antigen. ${ }^{2}$ These sera were examined in the IFAT at a dilution of 16 using both the polyvalent (serogroups 1-4) heat-killed antigen and the FYSA supplied by the Division of Microbiological Reagents and Quality Control at Colindale. Four sera were found to be positive at a dilution of 16 , in each case by both antigens. In view of this finding, we examined a further 500 sera on receipt with a serum dilution series starting at 16 . To our surprise we found, as shown in the Table, that only $13(2.7 \%)$ of the $\mathbf{4 8 3}$ sera from patients without a rising or high titre $(\geqslant 256)$ of antibody to $L$ pneumophila serogroups $1-4$ reacted to a titre of 16-128. These results are similar to those noted by Dr Taylor and Mr Harrison, but differ from those obtained by us over an earlier period (1978-80) when the proportion of sera from patients with titres falling in the range $32-128$ and not coming

from known cases ("known cases" wereํำ those with a rising titre of antibody or demonstration of Legionella infection b examination of tissue by direct immunofluorescence) was $13 \% .^{3}$ The reason for this finding is unknown as wके have used the same batch of antigen since 1979.

We now screen all sera for the detection of antigen to $L$ pneumophila serogroups $1-4$ starting at a dilution of 16 , in view of the sensitivity of the test and the predomi- $\rightarrow$ nance of $L$ pneumophila of serogroup 1 as the cause of legionellosis. Although, as Taylor and Harrison note, infection with $L^{\sigma}$ pneumophila of serogroups other than 1 is rare in their experience, we have reported that six of 67 patients with serologically

Numbers of sera reacting in the indirect fluorescent antibody test with polyvalent antigen prepared from $L$ pneumophila serogroups $1-4$

\begin{tabular}{llllll}
\hline Antibody titre & \multicolumn{5}{c}{ Total } \\
\hline$<16$ & 16 & 32 & 64 & 128 & \\
\hline 470 & 6 & $4^{*}$ & 2 & 1 & 483 \\
\hline
\end{tabular}

*These sera came from two patients. 
the clinical applications of such measurements.

The specific response of infection and allergy has of course been used for many years in one form or another for diagnosing and monitoring disease. However, it is surprising how little the sensitivity of radioimmunoassay has been exploited in this field. This book discusses the use of such assays for the management of allergy together with the benefits of in vitro measurement over skin testing. Examples are given of antibody measurement in various forms of infection such as extrinsic allergic alveolitis, brucellois and candidiasis allowing readers to see the wider potential in fields of their own interest. A brief discussion of immune complex assays is included though not in enough detail to be very useful. A brief but wide-ranging review of antibody measurement in general includes anti-idiotype and allotype antibodies, anti-tumour antibodies and antibodies to drugs. For those in non-medical fields a chapter on veterinary applications is included.

Overall a fascinating and useful book which certainly whets the appetite. It brings together ideas from diverse fields of pathology and should be of interest to clinical pathologist and researcher alike.

JT WHICHER

Cushing's Syndrome. Monographs on Endocrinology. Vol 22. DT Krieger. (Pp 142; illustrated; DM 88; US\$41.00.) Springer-Verlag. 1982.

Cushing's Syndrome is a distinguished addition to this series of monographs on endocrinology. Although it is not common in practice, the disentangling of the clinical enigma of overactivity of the adrenal cortex has and continues to yield information of wide biological interest. Dr. Krieger has herself made a signal contribution in this field.

The organisation of the book is conventional with chapters on the regulation of ACTH secretion (including reference to the isolation and structure of CRF reported in 1981) and ACTH action. Two thirds of the book, however, is concerned with clinical manifestations; investigation, and treatment of Cushing's syndrome. The emphasis is mostly on Cushing's disease and consideration of treatment of the more common condition of ectopic ACTH production is cursory. Nevertheless this is an admirable, up-to-date, and well referenced monograph.
The Pathology and Management of Thyroid Disease. Clinics in Endocrinology and Metabolism. Vol 10 No 2. Guest ed ED Williams. (Pp 161; illustrated; £9.75.) WB Saunders Company Ltd. 1981.

The general excellence of the "Clinics" monographs is due to the choice of wellknown and widely travelled editors who are personally acquainted with the specialists in their field. Experts usually agree to participate in a well conceived volume when they respect the editor. The additional advantage of the "Clinics" is their rapid publication. In this series only six months elapse between writing a review and its appearance in print, whereas most symposia and textbooks may linger for two to four years before they see the light of day. This slim volume is particularly helpful to any one involved in diagnosis or treatment of thyroid disorders because it deals succinctly with several controversial areas in thyroid pathology. Some chapters are written by surgeons and others by renowned pathologists and cytologists from Scandinavia, France, UK, and USA. Fine needle aspiration biopsy/cytology is well covered and needs to be taken up much more widely than it has been so far. The older method of large needle biopsy never became popular as it so often required general anaesthesia, which seemed unfair on the patient. Other controversies covered are the occurrence of malignancy in dyshormonogenetic goitres of after neck irradiation, thyroid inclusions in lymph nodes, the relationship between thyroid lymphomas and autoimmune thyroiditis, and the whole subject of medullary calcitonin tumours, sporadic and hereditary. The contributions are so attractively written and illustrated that reading this book truly combines business with pleasure.

DEBORAH DONIACH

\section{In the Technical method by Davies et al in the issue of July 1982, the source of reference 8 should read $J$ Pathol 1982;137:60. \\ Reference \\ ' Davies JD, Young EW. Congo blue: a rapid stain for elastic fibres. J Clin Pathol 1982;35:789. \\ Correction}

\section{Notices \\ International Symposium on Progress in Perinatal Medicine}

This symposium will be held in Florence, Italy, 10-12 May 1983. Topics will include: Perspective in fetal diagnosis; RIA in the antenatal evaluation of normal fetusproteins and hormones; methods for Toxoplasma Rubella, Viruses; Immune response of etus; Clinical diagnostic techniques and methods for congenital diseases. For information, please contact: A Albertini, Via Valsabbina, 1 Brescia, Italy.

\section{Heinz Karger Prizes}

The Heinz Karger Memorial Foundation invites the submission of papers on the following subjects:

1983: An original research paper or a review summarising an author's personal contributions to "cellular ageing". "Invasion and metastasis" (concerning either basic or clinical investigations in order to shed light on the process of malignant cell dissemination).

In 1983, the Foundation will commemorate its 20th anniversary by granting an award of Sw Fr 20000 . In 1984, the prize carries a award of Sw Fr 10000 . For further including rules for manuscript preparation) please contact: S Karger AG,
BASEL, Auschwilerstrasse $10, \mathrm{CH}-4009$ Basel.
1984: An original research paper on 